\title{
Demise of monocultural algal farms by exclusion of territorial damselfish
}

\author{
Hiroki Hata*, Makoto Kato \\ Graduate School of Human and Environmental Studies, Kyoto University, Sakyo, Kyoto 606-8501, Japan
}

\begin{abstract}
Resident herbivorous damselfishes exclude other herbivores from their territories and maintain dense stands of filamentous algae as algal farms. The damselfish Stegastes nigricans occupies coral reefs in the Okinawa Islands, southern Japan, and is unique in maintaining monocultural algal farms of the filamentous rhodophyte Polysiphonia sp. One of the mechanisms by which S. nigricans maintains these unique algal farms is by selective weeding of indigestible algae. This study tested the hypothesis that, were it not for management by $S$. nigricans, monocultures would be taken over by algal succession, even without grazing by other herbivores. We set up $S$. nigricans-exclusion cages inside and grazer-exclusion cages outside the territories of $S$. nigricans, and monitored algal succession. After exclusion of $S$. nigricans, the Polysiphonia sp. monoculture deteriorated rapidly and algal biomass decreased simultaneously. Filamentous rhodophytes replaced Polysiphonia sp. within $2 \mathrm{wk}$, and were in turn displaced by corticated macrophytes and calcareous algae (which are usually selectively weeded by $S$. nigricans). Monocultures of Polysiphonia sp. did not appear in the grazerexclusion cages; pioneer Polysiphonia sp. colonized the areas just after caging, but thereafter was replaced by other filamentous rhodophytes, corticated macrophytes, and calcareous algae. Our experiments demonstrated that $S$. nigricans maintained monocultures and high biomass of palatable pioneer Polysiphonia sp. against algal succession by intensive farm-management, involving selective weeding.
\end{abstract}

KEY WORDS: Algal farm management $\cdot$ Monoculture $\cdot$ Weeding $\cdot$ Stegastes nigricans $\cdot$ Polysiphonia sp. · Algal succession · Cage experiment

\section{INTRODUCTION}

Plant-herbivore interactions greatly affect the structure and function of ecosystems. In marine ecosystems, only a few host-specific interactions have been reported between algae and algivores (Steneck 1982, Hay et al. 1987, Hay 1991, John et al. 1992), and some unique plant-herbivore interactions have been noted between algae and potentially polyphagous algae-feeding fish. Resident herbivorous damselfishes exclude other herbivores from their territories, and maintain dense stands of filamentous algae (Vine 1974, Brawley \& Adey 1977, Lassuy 1980, Lobel 1980, Montgomery 1980a,b, Hixon \& Brostoff 1983, 1996, Sammarco 1983, Ruyter van Steveninck 1984, Kamura \& Choonhabandit 1986, Hinds \& Ballantine 1987, Russ 1987, Klumpp \& Polunin 1989,
Ferreira et al. 1998) known as an algal farm (Hata \& Nishihira 2002). Dusky farmerfish Stegastes nigricans occupies coral reefs in the Okinawa Islands, in southern Japan. This fish is thought to be unique in maintaining a virtual monoculture that is dominated by the filamentous rhodophyte Polysiphonia sp. 1, which was treated as Womersleyella setacea by Hata \& Kato (2002), Hata \& Nishihira (2002) and Hata et al. (2002). Other resident herbivorous damselfishes also maintain algal farms, but with high species diversity (Ceccarelli et al. 2001). Since Polysiphonia sp. 1 is found almost exclusively in S. nigricans territories, occurring quite rarely outside them, Polysiphonia sp. 1 and S. nigricans have been suggested to be mutualistic (Hata \& Kato 2002).

Under natural conditions, nearly monocultural algal communities, or colonial turfs, are formed when algae 
endure physical stress and moderate grazing pressure (Hay 1981, Sousa et al. 1981). These turf-forming algal species possess prostrate and upright branches, and exhibit considerable morphological plasticity in response to grazing. Compared with the algal species in colonial turfs, Polysiphonia sp. 1 is softer, has fewer chemical and physical defenses, and is competitively inferior.

One of the mechanisms by which Stegastes nigricans maintains these unique algal farms is by selective weeding of less digestible algae (Hata \& Kato 2002). Without selective weeding, an algal farm might be displaced by competitively superior algae. The aim of the present study was to verify the role of unique, intensive management used by $S$. nigricans to maintain the monocultural algal farms of Polysiphonia sp. 1. To confirm whether Polysiphonia sp. 1 monoculture would occur in the absence of $S$. nigricans management, we set up cages to exclude $S$. nigricans, within their territories, and monitored the succession of algal communities that grew inside the cages. We also set up grazerexclusion cages outside the territories of $S$. nigricans. The substrata outside the territories were exposed to intensive and continuous grazing pressure, and algal recruitment and growth were quite limited (Hata et al. 2002). Algal communities in these grazer-exclusion cages outside the territories were monitored to confirm whether the monoculture of Polysiphonia sp. 1 would occur in the absence of grazing and management by $S$. nigricans. By comparing the resulting algal communities in the cages with those observed in the territories of $S$. nigricans, we elucidated how monocultural algal farms are maintained.

\section{MATERIALS AND METHODS}

Study area. The study was carried out in the inner flat of a fringing reef on the west coast of Sesoko Island $\left(26^{\circ} 38^{\prime} \mathrm{N}, 127^{\circ} 52^{\prime} \mathrm{E}\right)$, in the NW part of Okinawa. We selected an area of about 50 by $200 \mathrm{~m}$ with similar wave action, water flow, and sedimentation (Yamanouchi 1993). In this area, grazers such as scarid fishes (e.g. Calotomus carolinus, Scarus sordidus, S. ghobban), acanthurid fishes (e.g. Acanthurus nigrofuscus, A. triostegus), siganid fishes (e.g. Siganus guttatus, S. spinus), and sea urchins (e.g. Diadema setosum, Echinometra spp., Tripneustes gratilla) are common.

Stegastes nigricans -exclusion experiment. Adult $S$. nigricans is about $84 \mathrm{~cm}$ in standard length and maintains about $2700 \mathrm{~cm}^{2}$ algal farm on coral rocks individually (Hata \& Kato unpubl.). Within the algal farm, exclusion cages were set on flat horizontal substrata and the succession of algal communities inside them were monitored. These cages were made of $20 \times$ $20 \mathrm{~mm}$ plastic mesh, which was sufficient to exclude $S$. nigricans, with a volume of $15 \times 15 \times 15 \mathrm{~cm}^{3}$. These cages served mainly for exclusion of the focal fish because within the territories, especially on flat horizontal substrata, all the herbivores are intensively excluded by the fish (Hata \& Kato unpubl.). We confirmed that no macroherbivore was found inside these cages and grazer-exclusion cages below. A total of 31 cages were placed within the territories of 31 adult S. nigricans on 29 May 2002 using stainless-anchor bolts embedded in holes drilled in the natural substratum on each corner. The cages were cleared of algae and other epibionts by brushing the mesh at least every 4 wk. At each timepoint thereafter $(1,2,4,8,12$, and $18 \mathrm{wk}$ ), 5 cages (or 6, in some cases) were removed, and quadrats $(7 \times 7 \mathrm{~cm})$ were immediately established in place of cages. All benthic algae, animals, and sediments in each quadrat were collected using a venturi suction sampler with a $120 \mu \mathrm{m}$ mesh bag (McShane \& Smith 1988, Hata et al. 2002). The mesh bags and their contents were preserved immediately after sampling in a $10 \%$ neutralized seawaterformalin mixture. All algae in the samples were identified to species level under a microscope, and the wet weight of each species was measured after removing excess water by blotting with a paper towel.

Grazer-exclusion experiment. We monitored algal succession in grazer-exclusion cages set outside the territories of Stegastes nigricans. These cages were made of $2 \times 2 \mathrm{~mm}$ panels of galvanized wire mesh, with a volume of $15 \times 15 \times 15 \mathrm{~cm}^{3}$. Outside the territories, herbivores of various sizes including schooling juvenile siganid fishes were present, and this mesh size was small enough to exclude all of them. Cages were cleared of algae and other epibionts by brushing the mesh at least every $4 \mathrm{wk}$. A total of 20 grazer-exclusion cages were set outside $S$. nigricans territories on flat horizontal surfaces of randomly selected coral rocks within the study area on 25 July 2002. At each timepoint thereafter $(1,2,4$, and $10 \mathrm{wk}), 5$ cages were removed, $7 \times 7 \mathrm{~cm}$ quadrats immediately established, algal samples were collected, and algae were identified and weighed, as described above for the S. nigricans-exclusion cages.

Cage controls. We used a shading structure (a roofonly cage without sides) to control for effects of decreased light levels in the cages (Sammarco 1983, Hixon \& Brostoff 1996). Roofs were constructed of $20 \times$ $20 \mathrm{~mm}$ mesh for the Stegastes nigricans-exclusion experiment and of $2 \times 2 \mathrm{~mm}$ mesh for the grazerexclusion experiment. The roof-only cages were set on flat horizontal surfaces of randomly selected coral rocks outside the territories on the same day as the respective exclusion cages. At each timepoint of 2, 8, 
and 12 wk (for the $S$. nigricans-exclusion controls) or 2 , 4 , and $10 \mathrm{wk}$ (for the grazer-exclusion controls), 5 roofonly cages were removed, $7 \times 7 \mathrm{~cm}$ quadrats immediately established, and algal samples were collected, and algae were identified and weighed, as described above for the $S$. nigricans-exclusion cages. At the same time, 5 quadrats were established outside the territories on flat horizontal surfaces of randomly selected coral rocks within the study area; algae within the quadrats were collected, identified, and weighed as described above. Algal communities were then compared between inside and outside the cages.

We also measured light intensity inside and outside both types of exclusion cage in situ on calm, cloud-free days at 09:00, 12:00, and 15:00 h ( $\mathrm{n}=12$ paired measurements per timepoint) using a LI-COR LI-1000 Data Logger. Relative water motion inside and outside both types of cage was also examined by measuring the mass loss of 7 pairs of plaster balls set inside and outside each cage (Komatsu \& Kawai 1992).

Data analysis. Algal succession was monitored using 4 indices (Hill 1973): (1) biomass; (2) species richness (number of species, $S$ ); (3) evenness (Pielou's $J_{i}$ Pielou 1966); and (4) species diversity (Shannon-Wiener index, $H^{\prime}$; Shannon \& Weaver 1949). Levene's test for homogeneity of variance yielded no significant results for all 4 indices. Therefore, to detect the effect of caging duration on these indices, 1-way analysis of variance (ANOVA) was used for analyses with the period of cage deployment as a fixed factor. Each of the 4 indices was compared among the periods using a multiple comparison test (least significant difference, LSD). Indices for the roof-only cages were compared with those for outside the cages using Mann-Whitney $U$-test. All analyses were conducted using the computer program SPSS for Windows.

Algal functional groups. Algal species were classified into 6 functional groups after Steneck \& Watling (1982): (1) microscopic filaments of cyanophytes (microalgae); (2) delicately branched filamentous algae with no or light cortices (filamentous algae); (3) thin tuber and foliose algae (foliose algae); (4) coarsely branched filamentous algae with cortices (corticated macrophytes); (5) thick blades with thick cortices (leathery macrophytes); and (6) calcified algae (calcareous algae). These functional groups correspond to life history strategies and competitive ability (Littler 1980, Littler et al. 1983a,b).

\section{RESULTS}

\section{Cage controls}

The Stegastes nigricans - exclusion cages decreased total light intensity by an average $( \pm 1$ SD) of $8.1 \pm$ $12.1 \%$ at $09: 00 \mathrm{~h}, 6.8 \pm 4.3 \%$ at $12: 00 \mathrm{~h}$, and $5.2 \pm 6.6 \%$ at $15: 00 \mathrm{~h}$ (Table 1). The grazer-exclusion cages also decreased total light intensity by an average ( $\pm 1 \mathrm{SD}$ ) of $15.6 \pm 13.7 \%$ at $09: 00 \mathrm{~h}, 13.6 \pm 12.9 \%$ at $12: 00 \mathrm{~h}$, and $23.3 \pm 5.6 \%$ at $15: 00 \mathrm{~h}$. Even inside these cages at 09:00 h, however, total light intensity was much greater than the level needed for saturation of the photosynthetic rate of a broad variety of algae (Lüning 1981, Littler \& Littler 1992, Kirk 1994). These results indicate that shading by cages was not important to algal succession (Hixson \& Brostoff 1996). Water motion differed significantly only inside the grazerexclusion cages compared with outside, although the cages reduced water motion by an average $( \pm 1$ SD) of only $8.1 \pm 5.1 \%$. No extra accumulation of sediments was observed inside both cages.

In algal farms, Polysiphonia sp. 1 strongly dominated throughout the year (Fig. 1A, Hata et al. 2002). We also conducted cage control experiments outside the territories to examine the secondary effect of caging. Outside cages, Polysiphonia sp. 1 was never found (Fig. 1B, Table 2). A mat-forming cyanophyte, Calothrix aeruginosa, and filamentous rhodophytes Herposiphonia obscura and Taenioma perpusillum composed algal communities with much lower biomass than in algal farms (for details, see Hata et al. 2002).

Table 1. Total light intensity inside and outside of Stegastes nigricans-exclusion cages $(20 \times 20 \mathrm{~mm}$ mesh) and grazer-exclusion cages $\left(2 \times 2 \mathrm{~mm}\right.$ mesh). Both $\mathrm{n}=12$. Comparisons were made using paired $t$-test: ${ }^{*} 0.05 \geq \mathrm{p} 0.01 ;{ }^{* *} 0.01 \geq \mathrm{p} 0.001 ;{ }^{* * *} \mathrm{p}<0.001$

\begin{tabular}{|c|c|c|c|c|c|c|}
\hline \multirow[b]{2}{*}{ Cage } & \multirow[b]{2}{*}{$\begin{array}{l}\text { Time } \\
(\mathrm{h})\end{array}$} & \multicolumn{2}{|c|}{ Inside cage -} & \multirow[b]{2}{*}{ Significance } & \multicolumn{2}{|c|}{ Outside cage } \\
\hline & & $\begin{array}{l}\text { Light intensity } \\
\left(\mu \mathrm{mol} \mathrm{m}{ }^{-2} \mathrm{~s}^{-1}\right)\end{array}$ & SD & & $\begin{array}{l}\text { Light intensity } \\
\left(\mu \mathrm{mol} \mathrm{m} \mathrm{m}^{-2} \mathrm{~s}^{-1}\right)\end{array}$ & $\mathrm{SD}$ \\
\hline \multirow{3}{*}{$\begin{array}{l}\text { S. nigricans } \\
\text { exclusion }\end{array}$} & 09:00 & 1484.7 & 170.1 & * & 1625.6 & 162.4 \\
\hline & $12: 00$ & 2560.8 & 257.5 & $* * *$ & 2750.0 & 281.4 \\
\hline & $15: 00$ & 1488.9 & 335.5 & * & 1568.6 & 316.6 \\
\hline \multirow{3}{*}{$\begin{array}{l}\text { Grazer } \\
\text { exclusion }\end{array}$} & 09:00 & 1460.1 & 196.9 & $* *$ & 1742.3 & 175.2 \\
\hline & $12: 00$ & 1755.1 & 158.6 & $* *$ & 2051.4 & 185.4 \\
\hline & $15: 00$ & 1483.3 & 166.1 & $* * *$ & 1944.5 & 250.3 \\
\hline
\end{tabular}



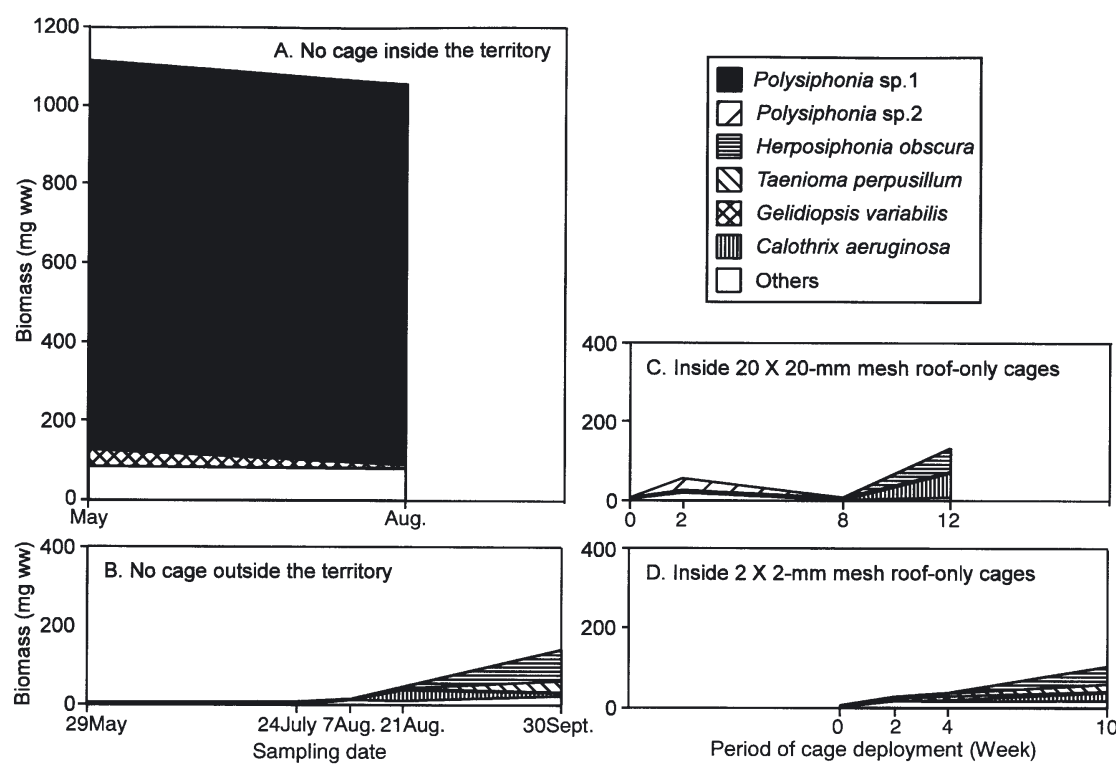

Fig. 1. Algal species composition (A) inside the territory of Stegastes nigricans ( $\mathrm{n}=18,16$ for May, August, respectively; Hata et al. 2002), (B) outside the territory $(\mathrm{n}=5)$, (C) inside $20 \times 20 \mathrm{~mm}$ mesh roof-only cages $(\mathrm{n}=5)$, and (D) inside $2 \times 2 \mathrm{~mm}$ mesh roof-only cages $(\mathrm{n}=5)$

These algae were early-colonizers and also constituted a large proportion of algal communities inside both roof-only cages (Fig. 1C,D, Table 2). Inside one $20 \times$ $20 \mathrm{~mm}$ mesh roof-only cage in the second week, Polysiphonia sp. 2, which also occurred outside cages, showed high biomass. This occasional increase of Polysiphonia sp. 2 resulted in significantly higher biomass and lower evenness in that cage in the second week than those outside the cages (Fig. 2). No other difference in all 4 indices was detected between inside both roof-only cages and outside the cages, and the algal communities inside both roof-only cages showed similar, slight seasonal changes to those outside cages.

These results indicated that the secondary effects of these cages were negligible compared with their primary effect of excluding either Stegastes nigricans or other grazers, and that under intensive grazing outside the territories, early-colonizing algae except Polysiphonia sp. 1 composed of algal communities with much lower biomass.

\section{Stegastes nigricans-exclusion experiment}

Inside Stegastes nigricans-exclusion cages, algal communities changed drastically during the study (Fig. 3A, Table 2). Before the cages were put in place, Polysiphonia sp. 1 strongly dominated (average relative abundance based on wet biomass $\pm 1 \mathrm{SD}=87.3 \pm$ $10.0 \%$; $=5$ ), but after the cages were placed, Polysiphonia sp. 1 declined quickly and was displaced by filamentous rhodophytes (Polysiphonia sp. 2 and Neosiphonia savatieri). Thereafter, a filamentous rhodophyte (Anotrichium tenue), corticated macrophytes (Gelidiella pannosa and Chondria sp.), and calcareous macrophytes (Jania arborescens and Bornetella sphaerica) increased in biomass. In terms of functional groups, filamentous algae were gradually replaced by corticated macrophytes and calcareous algae (Fig. 3B).

Algal biomass decreased initially, owing to the decrease of Polysiphonia sp. 1 biomass, and then increased due to the growth of the later-colonizing algae (Fig. 4A). Species richness increased quickly until the second week, because rare species started to inhabit the cages shortly after they were put in place (Fig. 4B, Table 2). Corresponding to the decline of Polysiphonia sp. 1, the evenness of the algal community increased rapidly until the fourth week after the cages started to operate, and then reached a plateau (Fig. 4C), as did species diversity (Fig. 4D).

After exclusion of Stegastes nigricans, the Polysiphonia sp. 1 monoculture deteriorated and was replaced by many competitively superior late-colonizing algae, and accordingly algal species diversity in the cages increased.

\section{Grazer-exclusion experiment}

Without grazing, the Polysiphonia sp. 1 monocultures seen in Stegastes nigricans algal farms were not present outside the territories of S. nigricans. In total, 33 algal species occurred inside the grazer-exclusion cages during the $10 \mathrm{wk}$ of the experiment (Table 2). Polysiphonia sp. 1 appeared in the first week and then disappeared (Fig. 5A). In succession, a filamentous rhodophyte (P. coacta), a cyanophyte microalga (Calothrix aeruginosa), and a calcareous chlorophyte (Acetabularia parvula) increased in biomass. By the tenth wk, another filamentous rhodophyte (Neosiphonia savatieri), corticated macrophytes (Gelidiopsis variabilis and Padina sp.), and a calcareous chlorophyte (Bornetella sphaerica and Rhipidosiphon javensis) had replaced them. From the standpoint of functional groups, filamentous algae colonized first, microalgae partially replaced them, and corticated macrophytes and calcareous algae came thereafter. Therefore, after $10 \mathrm{wk}$, these 4 algal groups were present in relatively equal amounts (Fig. 5B). 
Table 2. List of algal species and their abundance in the first 4 wk (F) and the rest of experimental periods (L) inside Stegastes nigricans-exclusion cages and inside grazer-exclusion cages, and cage controls conducted outside the territories, i.e. no cage, inside $20 \times 20 \mathrm{~mm}$ mesh and $2 \times 2 \mathrm{~mm}$ mesh roof-only cages. The temporal division was made because in the first $4 \mathrm{wk}$, caged algal communities changed drastically and were slightly saturated thereafter (see text for details). C: common occurrence more than $10 \%$ in relative biomass at least 1 sample; R: rare occurrence-less than $10 \%$ in relative biomass at every occurrence; -: no occurrence; Cy: Cyanophyta; Ch: Chlorophyta; He: Heterokontophyta; Rh: Rhodophyta

\begin{tabular}{|c|c|c|c|c|c|c|c|c|c|c|c|c|}
\hline \multirow{3}{*}{$\begin{array}{l}\text { Functional } \\
\text { groups }\end{array}$} & \multirow{3}{*}{ Species } & \multirow{3}{*}{ Phylum } & \multirow{2}{*}{\multicolumn{2}{|c|}{$\begin{array}{c}\text { S. nigricans } \\
\text { exclusion }\end{array}$}} & \multirow{2}{*}{\multicolumn{2}{|c|}{$\begin{array}{c}\text { Grazer } \\
\text { exclusion }\end{array}$}} & \multicolumn{2}{|c|}{ bundance } & \multirow{2}{*}{\multicolumn{2}{|c|}{$20 \times 20 \mathrm{~mm}$}} & \multirow{2}{*}{\multicolumn{2}{|c|}{$\begin{array}{c}2 \times 2 \mathrm{~mm} \\
\text { roof cages }\end{array}$}} \\
\hline & & & & & & & $\begin{array}{r}\mathrm{N} \\
\mathrm{Ca}\end{array}$ & & & & & \\
\hline & & & $\mathrm{F}$ & $\mathrm{L}$ & $\mathrm{F}$ & $\mathrm{L}$ & $\mathrm{F}$ & $\mathrm{L}$ & F & $\mathrm{L}$ & $\mathrm{F}$ & $\mathrm{L}$ \\
\hline \multirow{3}{*}{$\begin{array}{l}\text { Micro- } \\
\text { algae }\end{array}$} & Calothrix aeruginosa & Cy & $\mathrm{R}$ & $\mathrm{R}$ & $\mathrm{C}$ & $\mathrm{C}$ & $\mathrm{R}$ & $\mathrm{C}$ & $\mathrm{C}$ & $\mathrm{C}$ & $\mathrm{C}$ & $\mathrm{C}$ \\
\hline & Calothrix sp. & $\mathrm{Cy}$ & - & - & $\mathrm{C}$ & - & - & $\mathrm{C}$ & - & $\mathrm{R}$ & $\mathrm{C}$ & $\mathrm{R}$ \\
\hline & Lyngbya confervoides & Cy & - & $\mathrm{R}$ & $\mathrm{R}$ & - & - & - & - & - & - & - \\
\hline \multirow{15}{*}{$\begin{array}{l}\text { Filamentous } \\
\text { algae }\end{array}$} & Anotrichium tenue & $\mathrm{Rh}$ & $\mathrm{R}$ & $\mathrm{C}$ & $\mathrm{C}$ & - & - & $\mathrm{R}$ & $\mathrm{R}$ & - & $\mathrm{R}$ & $\mathrm{R}$ \\
\hline & Centroceras clavulatum & $\mathrm{Rh}$ & $\mathrm{R}$ & $\mathrm{R}$ & $\mathrm{C}$ & $\mathrm{R}$ & - & $\mathrm{R}$ & $\mathrm{C}$ & $\mathrm{R}$ & $\mathrm{C}$ & $\mathrm{R}$ \\
\hline & Ceramium sp. & $\mathrm{Rh}$ & $\mathrm{R}$ & $\mathrm{R}$ & - & - & - & - & - & - & - & - \\
\hline & Corallophila huysmansii & $\mathrm{Rh}$ & $\mathrm{C}$ & $\mathrm{C}$ & $\mathrm{R}$ & - & $\mathrm{R}$ & $\mathrm{R}$ & $\mathrm{R}$ & - & - & - \\
\hline & Herposiphonia obscura & $\mathrm{Rh}$ & $\mathrm{C}$ & $\mathrm{C}$ & $\mathrm{R}$ & $\mathrm{C}$ & $\mathrm{C}$ & $\mathrm{C}$ & $\mathrm{C}$ & $\mathrm{C}$ & $\mathrm{C}$ & $\mathrm{C}$ \\
\hline & Neosiphonia savatieri & $\mathrm{Rh}$ & $\mathrm{C}$ & $\mathrm{R}$ & $\mathrm{R}$ & $\mathrm{C}$ & $\mathrm{C}$ & $\mathrm{R}$ & $\mathrm{C}$ & $\mathrm{R}$ & - & $\mathrm{R}$ \\
\hline & Polysiphonia coacta & $\mathrm{Rh}$ & - & $\mathrm{R}$ & $\mathrm{C}$ & $\mathrm{C}$ & - & $\mathrm{C}$ & - & $\mathrm{R}$ & $\mathrm{C}$ & $\mathrm{C}$ \\
\hline & Polysiphonia sp. 1 & $\mathrm{Rh}$ & $\mathrm{C}$ & $\mathrm{C}$ & $\mathrm{C}$ & - & - & - & - & - & - & - \\
\hline & Polysiphonia sp. 2 & $\mathrm{Rh}$ & $\mathrm{C}$ & - & - & - & - & $\mathrm{R}$ & $\mathrm{C}$ & $\mathrm{R}$ & - & - \\
\hline & Polysiphonia sp. 3 & $\mathrm{Rh}$ & $\mathrm{R}$ & $\mathrm{R}$ & $\mathrm{C}$ & $\mathrm{R}$ & $\mathrm{C}$ & $\mathrm{C}$ & $\mathrm{C}$ & $\mathrm{C}$ & $\mathrm{C}$ & $\mathrm{R}$ \\
\hline & Taenioma perpusillum & $\mathrm{Rh}$ & $\mathrm{C}$ & $\mathrm{C}$ & $\mathrm{C}$ & $\mathrm{C}$ & $\mathrm{C}$ & $\mathrm{C}$ & $\mathrm{C}$ & $\mathrm{C}$ & $\mathrm{C}$ & $\mathrm{C}$ \\
\hline & Cladophora sp. & $\mathrm{Ch}$ & $\mathrm{R}$ & $\mathrm{R}$ & $\mathrm{R}$ & - & $\mathrm{C}$ & $\mathrm{C}$ & $\mathrm{C}$ & $\mathrm{R}$ & $\mathrm{R}$ & $\mathrm{R}$ \\
\hline & Pseudobryopsis sp. & $\mathrm{Ch}$ & - & $\mathrm{R}$ & - & - & - & - & - & - & - & - \\
\hline & Struvea sp. & $\mathrm{Ch}$ & $\mathrm{R}$ & $\mathrm{R}$ & - & - & - & - & - & - & - & - \\
\hline & Feldmannia indica & $\mathrm{He}$ & $\mathrm{R}$ & $\mathrm{R}$ & $\mathrm{C}$ & $\mathrm{C}$ & $\mathrm{C}$ & $\mathrm{C}$ & $\mathrm{C}$ & $\mathrm{C}$ & $\mathrm{C}$ & $\mathrm{C}$ \\
\hline \multirow{3}{*}{$\begin{array}{l}\text { Foliose } \\
\text { algae }\end{array}$} & Dictyosphaeria versluysii & $\mathrm{Ch}$ & $\mathrm{R}$ & - & - & - & - & - & $\mathrm{C}$ & - & - & - \\
\hline & Enteromorpha sp. & $\mathrm{Ch}$ & $\mathrm{R}$ & $\mathrm{R}$ & - & - & $\mathrm{C}$ & $\mathrm{R}$ & $\mathrm{C}$ & $\mathrm{R}$ & $\mathrm{R}$ & - \\
\hline & Dictyota friabilis & $\mathrm{He}$ & - & - & - & $\mathrm{R}$ & - & - & - & - & - & - \\
\hline \multirow{11}{*}{$\begin{array}{l}\text { Corticated } \\
\text { macrophytes }\end{array}$} & Champia sp. & $\mathrm{Rh}$ & $\mathrm{R}$ & $\mathrm{R}$ & $\mathrm{R}$ & $\mathrm{R}$ & $\mathrm{C}$ & $\mathrm{R}$ & $\mathrm{C}$ & - & $\mathrm{R}$ & $\mathrm{R}$ \\
\hline & Chondria sp. & $\mathrm{Rh}$ & $\mathrm{R}$ & $\mathrm{C}$ & $\mathrm{R}$ & - & $\mathrm{C}$ & - & $\mathrm{C}$ & - & $\mathrm{R}$ & $\mathrm{R}$ \\
\hline & Gelidiella pannosa & $\mathrm{Rh}$ & $\mathrm{C}$ & $\mathrm{C}$ & $\mathrm{C}$ & $\mathrm{R}$ & $\mathrm{C}$ & $\mathrm{C}$ & $\mathrm{C}$ & $\mathrm{C}$ & $\mathrm{R}$ & - \\
\hline & Gelidiopsis variabilis & $\mathrm{Rh}$ & $\mathrm{C}$ & $\mathrm{C}$ & $\mathrm{C}$ & $\mathrm{C}$ & $\mathrm{C}$ & $\mathrm{C}$ & $\mathrm{C}$ & $\mathrm{C}$ & $\mathrm{C}$ & $\mathrm{R}$ \\
\hline & Hypnea sp. & $\mathrm{Rh}$ & - & $\mathrm{R}$ & - & - & - & $\mathrm{R}$ & - & - & $\mathrm{R}$ & - \\
\hline & Wrangelia sp. & $\mathrm{Rh}$ & $\mathrm{R}$ & $\mathrm{C}$ & - & $\mathrm{R}$ & - & - & - & - & - & - \\
\hline & Caulerpa ambigua & $\mathrm{Ch}$ & $\mathrm{R}$ & - & - & - & - & - & - & - & - & - \\
\hline & Caulerpa brachypus & $\mathrm{Ch}$ & - & - & - & $\mathrm{R}$ & - & - & - & - & - & - \\
\hline & Caulerpa serrulata & $\mathrm{Ch}$ & $\mathrm{R}$ & $\mathrm{R}$ & - & - & - & - & - & - & - & - \\
\hline & Caulerpa verticillata & $\mathrm{Ch}$ & $\mathrm{R}$ & $\mathrm{R}$ & - & - & - & - & - & - & - & - \\
\hline & Padina sp. & $\mathrm{He}$ & $\mathrm{R}$ & $\mathrm{R}$ & $\mathrm{C}$ & $\mathrm{C}$ & - & $\mathrm{C}$ & $\mathrm{R}$ & $\mathrm{R}$ & $\mathrm{C}$ & $\mathrm{R}$ \\
\hline \multicolumn{13}{|l|}{ Leathery } \\
\hline macrophytes & Sargassum sp. & $\mathrm{He}$ & - & - & $\mathrm{R}$ & $\mathrm{R}$ & - & $\mathrm{R}$ & - & - & - & $\mathrm{R}$ \\
\hline \multirow{12}{*}{$\begin{array}{l}\text { Calcareous } \\
\text { algae }\end{array}$} & Jania adhaerens & $\mathrm{Rh}$ & $\mathrm{R}$ & $\mathrm{C}$ & $\mathrm{C}$ & $\mathrm{R}$ & $\mathrm{C}$ & $\mathrm{C}$ & $\mathrm{C}$ & $\mathrm{C}$ & $\mathrm{C}$ & - \\
\hline & Jania arborescens & $\mathrm{Rh}$ & $\mathrm{R}$ & $\mathrm{C}$ & - & $\mathrm{R}$ & - & - & - & - & - & - \\
\hline & Jania sp. & $\mathrm{Rh}$ & - & $\mathrm{R}$ & - & - & - & - & - & - & - & - \\
\hline & Acetabularia clavata & $\mathrm{Ch}$ & $\mathrm{R}$ & $\mathrm{R}$ & $\mathrm{R}$ & $\mathrm{R}$ & - & $\mathrm{R}$ & - & $\mathrm{R}$ & $\mathrm{R}$ & $\mathrm{R}$ \\
\hline & Acetabularia dentata & $\mathrm{Ch}$ & $\mathrm{R}$ & - & $\mathrm{R}$ & - & - & - & $\mathrm{R}$ & - & - & - \\
\hline & Acetabularia exigua & $\mathrm{Ch}$ & $\mathrm{R}$ & $\mathrm{R}$ & $\mathrm{C}$ & $\mathrm{R}$ & $\mathrm{C}$ & $\mathrm{R}$ & $\mathrm{C}$ & $\mathrm{C}$ & $\mathrm{R}$ & $\mathrm{R}$ \\
\hline & Acetabularia parvula & $\mathrm{Ch}$ & $\mathrm{R}$ & $\mathrm{R}$ & $\mathrm{C}$ & $\mathrm{C}$ & - & $\mathrm{R}$ & $\mathrm{C}$ & $\mathrm{C}$ & $\mathrm{R}$ & $\mathrm{R}$ \\
\hline & Bornetella sphaerica & $\mathrm{Ch}$ & $\mathrm{R}$ & $\mathrm{R}$ & $\mathrm{R}$ & $\mathrm{C}$ & - & - & $\mathrm{R}$ & $\mathrm{R}$ & - & $\mathrm{R}$ \\
\hline & Halimeda discoidea & $\mathrm{Ch}$ & - & $\mathrm{R}$ & - & - & - & - & - & - & - & - \\
\hline & Neomeris annulata & $\mathrm{Ch}$ & $\mathrm{R}$ & $\mathrm{R}$ & - & $\mathrm{R}$ & - & - & - & - & - & - \\
\hline & Penicillus sibogae & $\mathrm{Ch}$ & $\mathrm{R}$ & $\mathrm{R}$ & $\mathrm{R}$ & $\mathrm{R}$ & - & $\mathrm{R}$ & $\mathrm{R}$ & $\mathrm{C}$ & $\mathrm{R}$ & - \\
\hline & Rhipidosiphon javensis & $\mathrm{Ch}$ & $\mathrm{R}$ & $\mathrm{C}$ & - & $\mathrm{C}$ & - & $\mathrm{R}$ & - & - & - & - \\
\hline
\end{tabular}



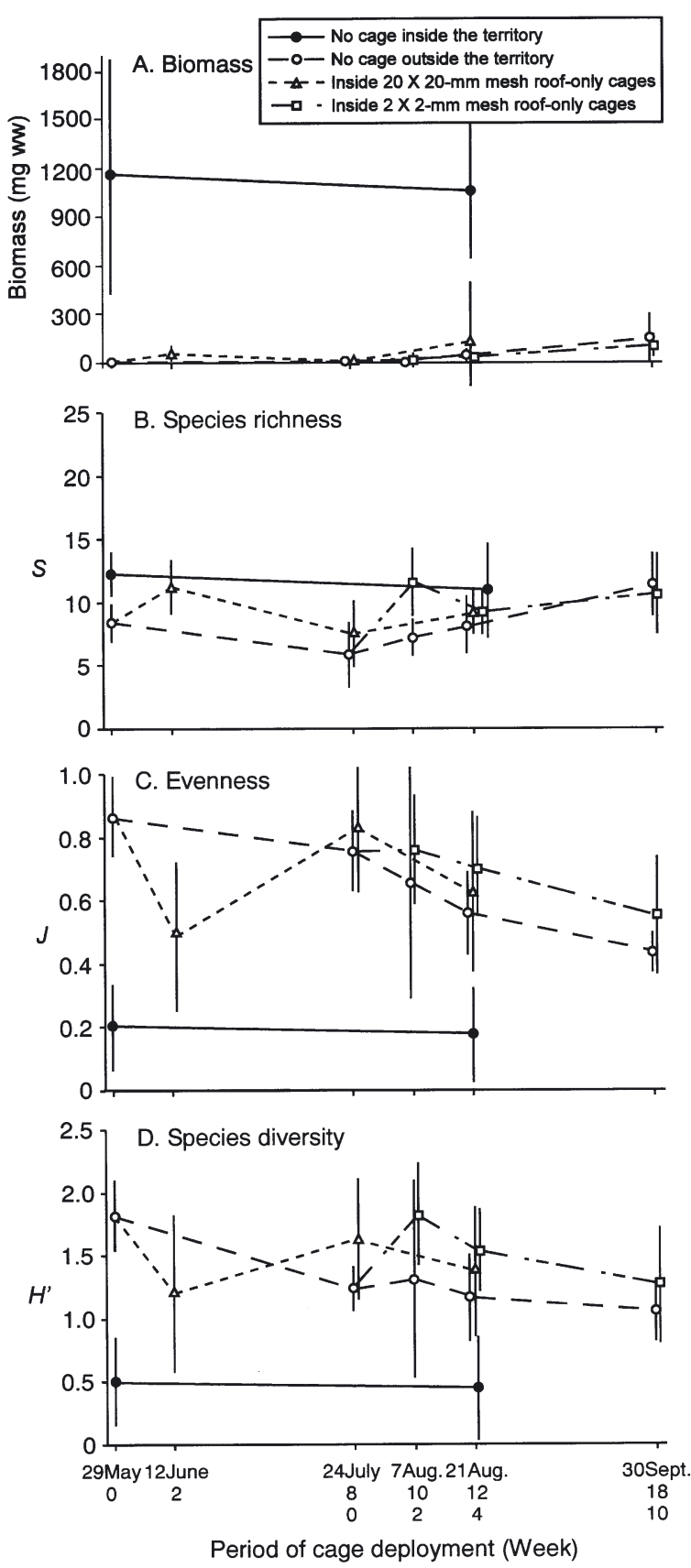

Fig. 2. Temporal patterns of (A) total algal biomass, (B) species richness, (C) evenness, and (D) species diversity for cage controls, i.e. no cage inside the territory (Hata et al. 2002), no cage outside the territory, inside $20 \times 20 \mathrm{~mm}$ mesh roof-only cages, and inside $2 \times 2 \mathrm{~mm}$ mesh roof-only cages. Each point represents the average $( \pm 1 \mathrm{SD})$ of $7 \times 7 \mathrm{~cm}$ quadrat samples. See Fig. 1 for sample sizes

In the grazer-exclusion cages, algal biomass increased quickly until after 4 wk of exclusion (Fig. 6A). Total algal biomass at Week 10 was still much lower than that in the Stegastes nigricans - exclusion cages. In the cages, algal species richness increased abruptly in the first wk and became nearly saturated (Fig. 6B). At Week 10, species richness was $15.0 \pm 2.3$ (average $\pm 1 \mathrm{SD}$ ), and this value was similar to that inside $S$. nigricans-exclusion cages after $18 \mathrm{wk}$ (16.4 $\pm 3.6 ; \mathrm{p}>0.05$, Mann-Whitney $U$-test). Evenness decreased during the first $4 \mathrm{wk}$ and increased thereafter (Fig. 6C). Because of high species richness and evenness, species diversity after $10 \mathrm{wk}$ was higher in the grazer-exclusion cages $(1.78 \pm 0.22)$ than in the algal farms managed by $S$. nigricans $(0.52 \pm 0.33 ; \mathrm{p}<$ 0.01 , Mann-Whitney $U$-test), and was also higher than inside the $S$. nigricans-exclusion cages at $18 \mathrm{wk}(1.22 \pm$ 0.37; p < 0.05, Mann-Whitney U-test; Fig. 6D). Compared to the algal assemblages outside cages, evenness and species diversity were higher in the cages at Wk 10 (both $\mathrm{p}<0.05$, Mann-Whitney $U$-test), although biomass and species richness were not significantly different between inside and outside the cages.

These results indicate that algal communities in the grazer-exclusion cages at Week 10 were composed of both early- and late-colonizing algae, and were in the mid-successional stage with high species diversity.

\section{DISCUSSION}

After exclusion of Stegastes nigricans, Polysiphonia sp. 1 monocultures deteriorated rapidly. Filamentous rhodophytes replaced Polysiphonia sp. 1 initially, fol-

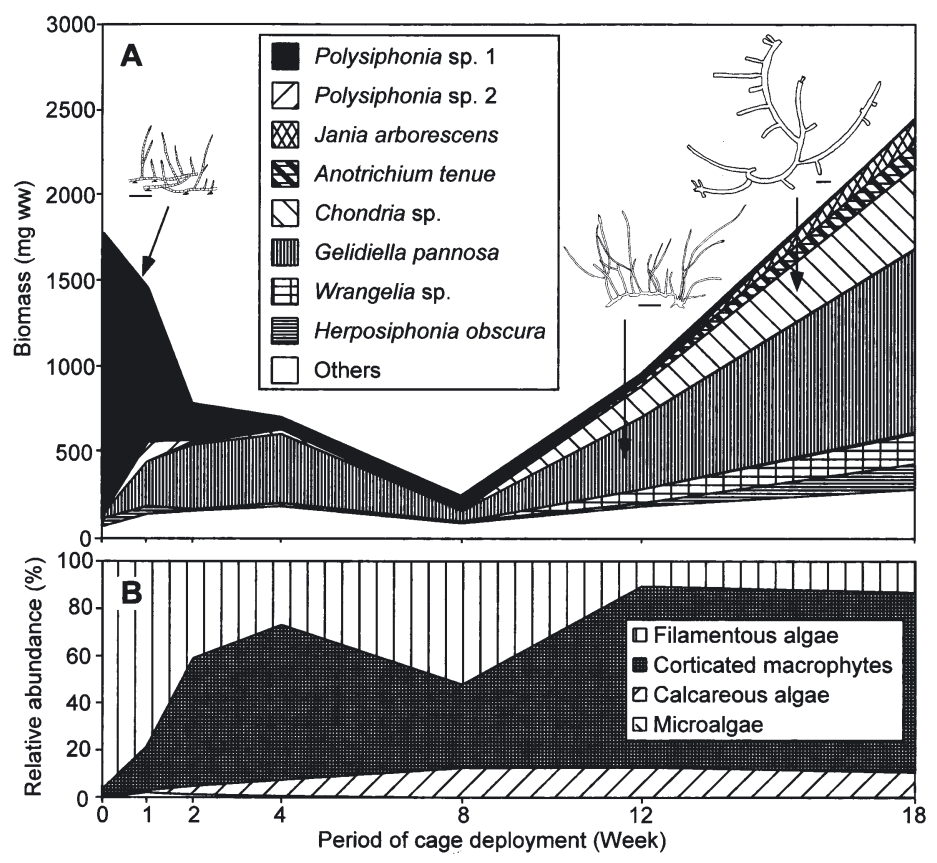

Fig. 3. (A) Algal species composition (scale bar = $1 \mathrm{~mm}$ ) and (B) relative abundance of wet biomass of algal functional groups inside the Stegastes nigricans-exclusion cages. Values are averages of 5 to 6 quadrat samples $(7 \times 7 \mathrm{~cm})$ per occasion 

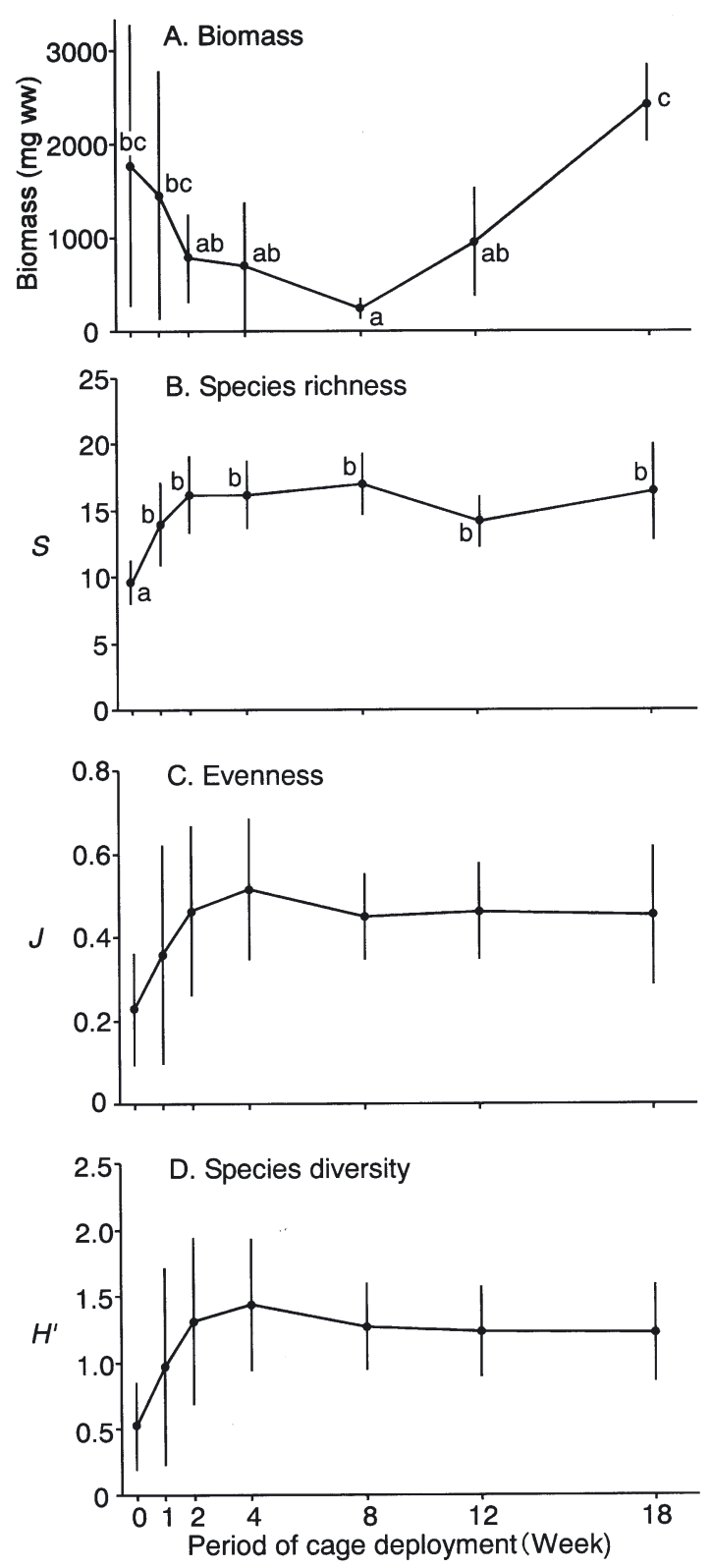

Fig. 4. Temporal patterns of (A) algal biomass, (B) algal species richness, (C) evenness, and (D) species diversity inside the Stegastes nigricans-exclusion cages. Each point represents the average $( \pm 1 \mathrm{SD})$ of 5 to 6 quadrat samples $(7 \times$ $7 \mathrm{~cm}$ ). Different letters indicate significant differences at the $5 \%$ level by 1 -way ANOVA followed by LSD test

lowed by the increase and domination of other filamentous rhodophytes, corticated macrophytes, and calcareous algae. Algal functional groups are strongly related to survival strategies and competitive ability (Littler \& Littler 1980, Littler et al. 1983a,b); microalgae and filamentous algae are early colonizers with high growth rates, while corticated macrophytes and calcareous algae are late-successional species with lower

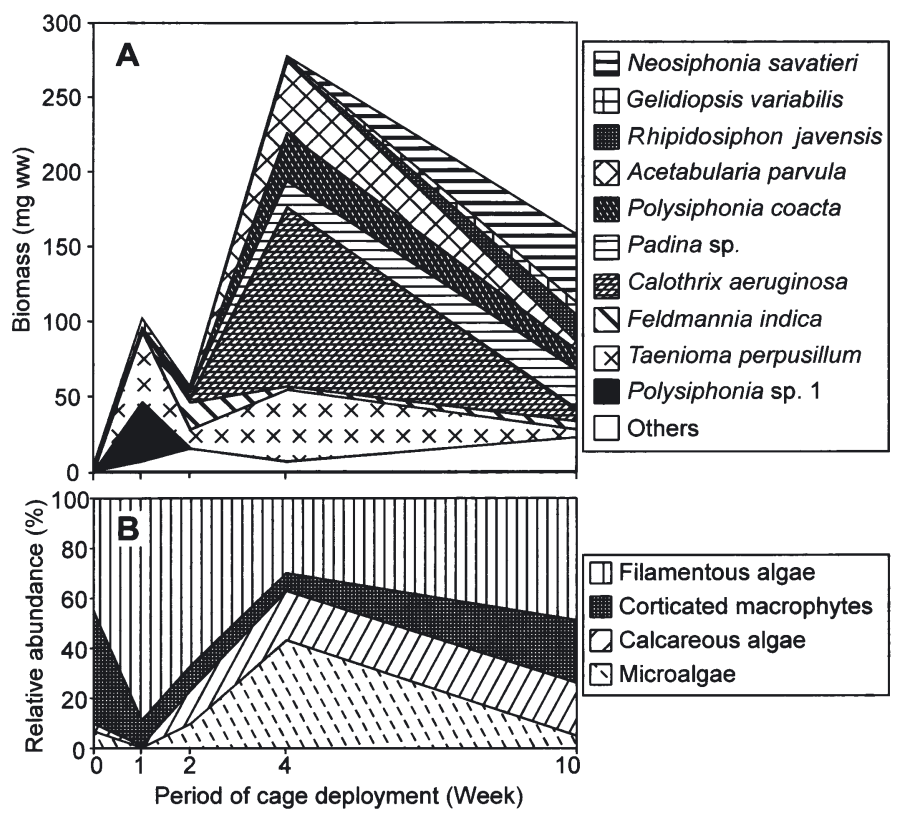

Fig. 5. (A) Algal species composition and (B) relative abundance of algal functional groups based on wet biomass inside the grazer-exclusion cages. Values are averages of 5 to 6 quadrat samples $(7 \times 7 \mathrm{~cm})$

growth rates and high competitive ability. Under grazing-free conditions inside the grazer-exclusion cages, the pioneering Polysiphonia sp. 1 was replaced by other filamentous rhodophytes, late-colonizing corticated macrophytes and calcareous algae, indicating an inferior competitive ability of Polysiphonia sp. 1. Furthermore, late-colonizing corticated macrophytes and calcareous algae that became dominant after $8 \mathrm{wk}$ in the $S$. nigricans-exclusion cages (e.g. corticated Gelidiella pannosa and Chondria sp., and calcareous Jania arborescens and Bornetella sphaerica) are not easily digested by $S$. nigricans and are selectively weeded (Hata \& Kato 2002). These results demonstrate that by intensive management involving selective weeding, $S$. nigricans manages algal farm monoculture of the palatable alga Polysiphonia sp. 1, and this alga may therefore reach high biomass values.

Algal farms of most other damselfishes are characterized by a high species-diversity of mid-successional algal communities, which are composed of both pioneers and late-colonizers (Ceccarelli et al. 2001). Other experiments involving exclusion of other territorial damselfishes (Lassuy 1980, Hinds \& Ballantine 1987) found that algal species diversity in cages set inside algal farms decreased because 1 or a few competitively superior alga species outcompeted and dominated algal communities that initially had high species diversity. The reported algal communities resembled those formed inside the grazer-exclusion cages at Wk 10 of 

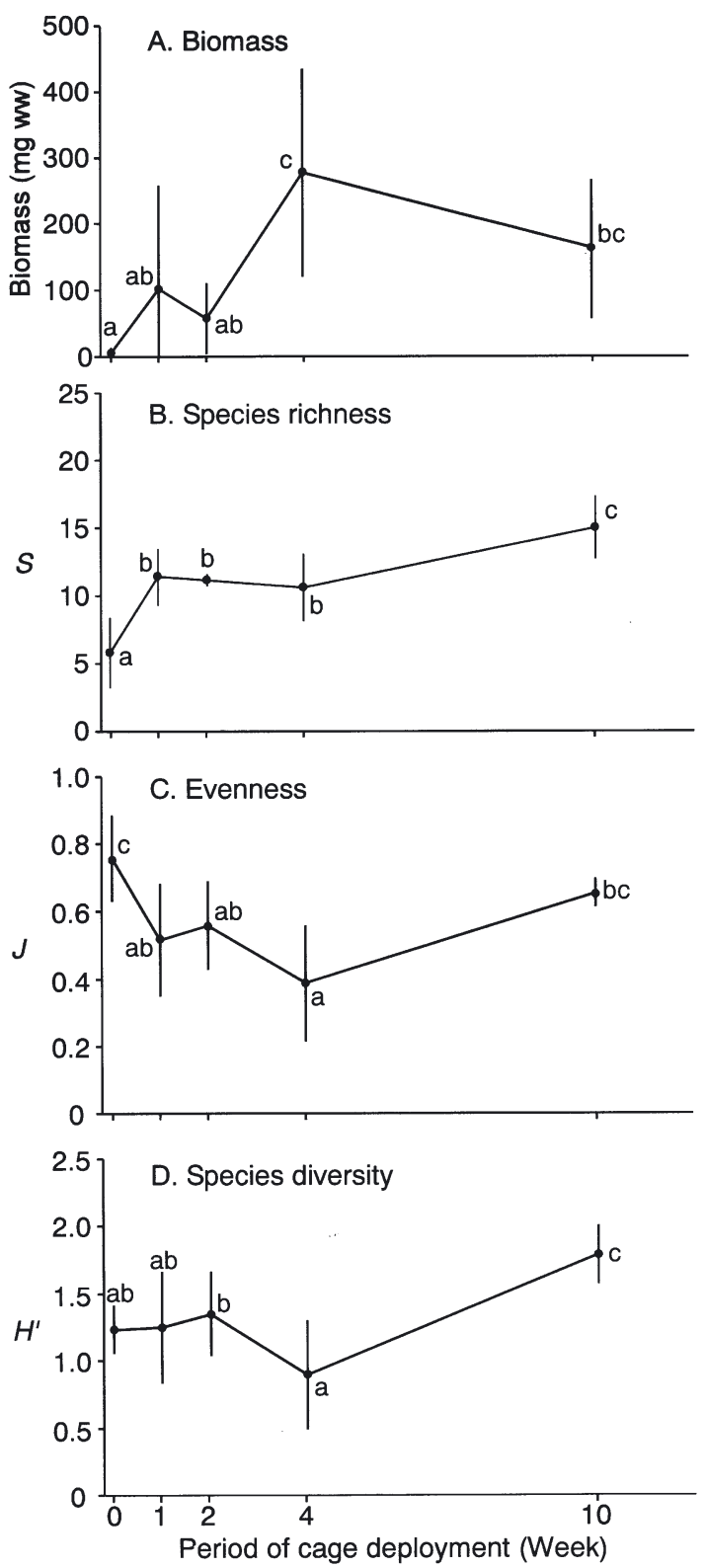

Fig. 6. Temporal patterns of (A) total algal biomass, (B) species richness, (C) evenness, and (D) species diversity inside the grazer-exclusion cages. Each point represents the average $( \pm 1 \mathrm{SD})$ of five $7 \times 7 \mathrm{~cm}$ quadrat samples. Different letters indicate significant differences at the $5 \%$ level by 1 -way ANOVA followed by LSD test

our experiment. In our Stegastes nigricans-exclusion experiment, the species-diversity of the algal communities within the cages increased. This discrepancy with the results of previous studies was caused by the initial difference in algal composition and suggests that the Polysiphonia sp. 1 monocultures with high biomass are established under conditions of halted succession due to intensive management, i.e. weeding of later-colonizers strongly prevents them from invading monoculture algal farms. Algal farms of other damselfishes, on the other hand, are maintained by slowed succession due to extensive management, i.e. keeping moderate grazing-pressure prevents later-colonizers from excluding early colonizers competitively (Hixon \& Brostoff 1996). In the algal farms of Hemiglyphidodon plagiometopon, species diversity increased when the fish were excluded by cages (Lassuy 1980). In these farms, the corticated rhodophyte Gelidiopsis intricata and the filamentous rhodophyte Polysiphonia scopulorum comprised $80 \%$ of the biomass; the algal species diversity was intermediate between that of the farms of $S$. nigricans and those of other damselfishes. These data suggest that farm management by $H$. plagiometopon may be intermediate, involving only some weeding.

In conclusion, Stegastes nigricans directly affects algal succession by its intensive management involving selective weeding on indigestible and late-colonizing algae to maintain highly digestible Polysiphonia sp. 1 monocultures in their algal farms. These results suggest that $S$. nigricans manage their algal farms much more intensively than other damselfishes, and that the relationship between Polysiphonia sp. 1 and $S$. nigricans is a new example of species-specific mutual interactions between plant and herbivore in marine ecosystems, where such relationships are quite rare.

Acknowledgements. We thank M. Nishihira for his instruction throughout our study, and S. Kamura and K. Kishimoto for their significant help with identification of algae. Thanks are due to K. Iwasaki for his suggestion about cage experiments. We are grateful to A. Nishikawa and K. Aoki for their kind help with our field experiments. We are also grateful to the referees for their significant comments. Sesoko Marine Science Center (SMSC), University of the Ryukyus provided the facilities for the field research and laboratory. This work was partly supported by the Sasakawa Scientific Research Grant from the Japan Science Society, and was also supported by JSPS Research Fellowships for Young Scientists.

\section{LITERATURE CITED}

Brawley SH, Adey WH (1977) Territorial behavior of threespot damselfish Eupomacentrus planifrons increases reef algal biomass and productivity. Environ Biol Fishes 2: 45-51

Ceccarelli DM, Jones GP, McCook LJ (2001) Territorial damselfishes as determinants of the structure of benthic communities on coral reefs. Oceanogr Mar Biol Annu Rev 39:355-389

Ferreira CE, Gonçalves JE, Coutinho R, Peret AC (1998) Herbivory by the dusky damselfish Stegastes fuscus (Cuvier, 1830 ) in a tropical rocky shore: effects on the benthic community. J Exp Mar Biol Ecol 229:241-264

Hata H, Kato M (2002) Weeding by the herbivorous damselfish Stegastes nigricans in nearly monocultural algae farms. Mar Ecol Prog Ser 237:227-231 
Hata H, Nishihira M (2002) Territorial damselfish enhances multi-species co-existence of foraminifera mediated by biotic habitat structuring. J Exp Mar Biol Ecol 270: $215-240$

Hata H, Nishihira M, Kamura S (2002) Effects of habitatconditioning by the damselfish Stegastes nigricans (Lacepède) on the community structure of benthic algae. J Exp Mar Biol Ecol 280:95-116

Hay ME (1981) The functional morphology of turf-forming seaweeds: persistence in stressful marine habitats. Ecology 62:739-750

Hay ME (1991) Marine-terrestrial contrasts in the ecology of plant chemical defenses against herbivores. Trends Ecol Evol 6:362-365

Hay ME, Fenical W, Gustafson K (1987) Chemical defense against diverse coral-reef herbivores. Ecology 68:1581-1591

Hill MO (1973) Diversity and evenness: a unifying notation and its consequences. Ecology 54:427-432

Hinds PA, Ballantine DL (1987) Effects of the Caribbean threespot damselfish, Stegastes planifrons (Cuvier), on algal lawn. Aquat Bot 27:299-308

Hixon MA, Brostoff WN (1983) Damselfish as keystone species in reverse: intermediate disturbance and diversity of reef algae. Science 220:511-513

Hixon MA, Brostoff WN (1996) Succession and herbivory: effects of differential fish grazing on Hawaiian coral-reef algae. Ecol Monogr 66:67-90

John D, Hawins SJ, Price JH (1992) Plant-animal interactions in the marine benthos. Clarendon Press, Oxford

Kamura S, Choonhabandit S (1986) Algal communities within territories of the damselfish Stegastes apicalis and the effects of grazing by the sea urchin Diadema spp. in the Gulf of Thailand. Galaxea 5:175-194

Kirk JTO (1994) Photosynthesis as a function of the incident light. In: Kirk JTO (ed) Light and photosynthesis in aquatic ecosystems. Cambridge University Press, Cambridge, p 271-313

Klumpp DW, Polunin NVC (1989) Partitioning among grazers of food resources within damselfish territories on a coral reef. J Exp Mar Biol Ecol 125:145-170

Komatsu T, Kawai H (1992) Measurements of time-averaged intensity of water motion with plaster balls. J Oceanogr 48:353-365

Lassuy DR (1980) Effects of 'farming' behavior by Eupomacentrus lividus and Hemiglyphidodon plagiometopon on algal community structure. Bull Mar Sci 30:304-312

Littler MM (1980) Morphological form and photosynthetic performances of marine macroalgae: tests of a functional/ form hypothesis. Bot Mar 22:161-165

Littler MM, Littler DS (1980) The evolution of thallus form and survival strategies in benthic marine macroalgae: field and laboratory tests of a functional form model. Am Nat 116:25-44

Littler MM, Littler DS, Taylor PR (1983a) Evolutionary strate-

Editorial responsibility: Otto Kinne (Editor),

Oldendorf/Luhe, Germany gies in a tropical barrier reef system: functional-form groups of marine macroalgae. J Phycol 19:229-237

Littler MM, Taylor PR, Littler DS (1983b) Algal resistance to herbivory on a Caribbean barrier reef. Coral Reefs 2: 111-118

Littler MM, Littler DS (1992) Photosynthesis vs. irradiance curves for six species of macroalgae from the Seychells Islands under four levels of nutrient enrichment. Atoll Res Bull 374:1-14

Lobel PS (1980) Herbivory by damselfishes and their role in coral reef community ecology. Bull Mar Sci 30:273-289

Lüning K (1981) Light. In: Lobban CS, Wynne MJ (eds) The biology of seaweeds. Blackwell Scientific, Oxford, p 326-355

McShane PE, Smith MG (1988) Measuring abundance of juvenile abalone, Haliotis rubra Leach (Gastropoda: Haliotidae); comparison of a novel method with two other methods. Aust J Mar Freshw Res 39:331-336

Montgomery WL (1980a) Comparative feeding ecology of two herbivorous damselfishes (Pomacentridae: Teleostei) from the Gulf of California, Mexico. J Exp Mar Biol Ecol 47:9-24

Montgomery WL (1980b) The impact of non-selective grazing by the giant blue damselfish, Microspathodon dorsalis, on algal communities in the Gulf of California, Mexico. Bull Mar Sci 30:290-303

Pielou EC (1966) The measurement of diversity in different types of biological collections. J Theor Biol 10:131-144

Russ GR (1987) Is rate of removal of algae by grazers reduced inside territories of tropical damselfishes? J Exp Mar Biol Ecol 110:1-17

Ruyter van Steveninck ED (1984) The composition of algal vegetation in and outside damselfish territories on a Florida reef. Aquat Bot 20:11-19

Sammarco PW (1983) Effects of fish grazing and damselfish territoriality on coral reef algae. I. Algal community structure. Mar Ecol Prog Ser 13:1-14

Shannon CE, Weaver W (1949) The mathematical theory of communication. University of Illinois Press, Chicago

Sousa WP, Schroeter SC, Gaines SD (1981) Latitudinal variation in intertidal algal community structure: the influence of grazing and vegetative propagation. Oecologia 48: 297-307

Steneck RS (1982) A limpet-coralline alga association: adaptations and defenses between a selective herbivore and its prey. Ecology 63:507-522

Steneck RS, Watling L (1982) Feeding capabilities and limitation of herbivorous molluscs: a functional group approach. Mar Biol 68:299-319

Vine PJ (1974) Effects of algal grazing and aggressive behaviour of the fishes Pomacentrus lividus and Acanthurus sohal on coral-reef ecology. Mar Biol 24:131-136

Yamanouchi H (1993) Sandy sediments on the coral reef and beach of northwest Sesoko Island, Okinawa. Galaxea 11: 107-133

Submitted: April 16, 2003; Accepted: September 7, 2003

Proofs received from author(s): November 18, 2003 